\title{
Upper Tract Urothelial Carcinoma: A Clinicopathologic Study Including Microsatellite Instability Analysis
}

\author{
Hagen Blaszyk, M.D., Linan Wang, M.D., Wolfgang Dietmaier, Ph.D., Ferdinand Hofstädter, M.D., \\ Lawrence J. Burgart, M.D., John C. Cheville, M.D., Arndt Hartmann, M.D. \\ Department of Laboratory Medicine and Pathology, Mayo Clinic and Foundation, Rochester, Minnesota \\ (HB, LW, LJB, JCC); and Institute of Pathology, University of Regensburg, Regensburg, Germany (HB, WD, \\ $\mathrm{FH}, \mathrm{AH})$
}

Urothelial carcinoma of the renal pelvis and ureter may develop as a manifestation of the hereditary nonpolyposis colorectal cancer syndrome that is characterized by mutations in a number of DNA mismatch repair genes and detectable as microsatellite instability. In this study, we examined microsatellite instability and the clinicopathologic features of urothelial carcinoma of the renal pelvis ( $n$ $=61)$ and ureter $(n=53)$ from 114 consecutive patients surgically treated from 1985-1992. Clinical data were obtained through chart review. Matched normal and tumor DNA was extracted from paraffin-embedded tissue, and a panel of six microsatellite loci was analyzed. The male-female ratio was 2.8:1 with a median age of 70 years (range, 28 to 92 y). Microsatellite analysis was successful in 67 tumors, and 21 (31.3\%) patients had tumors that exhibited microsatellite instability. Patients with microsatellite-unstable tumors were significantly more likely to have additional nonurologic cancers $(P=.015)$ including colorectal carcinoma $(P=.001)$ compared with patients with tumors that did not exhibit microsatellite instability. In addition, patients with microsatellite-unstable tumors showed more colorectal cancers in their family $(P=.026)$ and were more likely to have higher grade urothelial carcinoma of the upper tract $(P=\mathbf{. 0 2 8})$. Grade and stage, but not microsatellite status, were the strongest predictors of cancer-specific survival. This study found the highest frequency of microsatellite instability in upper urothelial tract carcinomas reported to date and highlights upper tract urothelial carcinoma as a marker of the hereditary nonpol-

Copyright (C) 2002 by The United States and Canadian Academy of Pathology, Inc.

VOL. 15, NO. 8, P. 790, 2002 Printed in the U.S.A.

Date of acceptance: May 1, 2002.

Address reprint requests to: Hagen Blaszyk, M.D., Department of Pathology, University of Regensburg Medical Center, PF 1006 42, D-93042 Regensburg, Germany; e-mail: hagen.blaszyk@klinik.uni-regensburg.de; fax: 49-941-944-6602

DOI: 10.1097/01.MP.0000024263.25043.0C yposis colorectal cancer syndrome in some patients. These findings reinforce the importance of obtaining cancer histories in patients with upper tract urothelial carcinoma to subsequently identify individuals with the hereditary nonpolyposis colorectal cancer syndrome and at-risk relatives for surveillance and management programs.

KEY WORDS: Family history, HNPCC, Microsatellites, Renal pelvis, Smoking, Ureter, Urothelial carcinoma,

Mod Pathol 2002;15(8):790-797

Urothelial carcinoma of the upper urinary tract (renal pelvis and ureter) is relatively uncommon, representing $5 \%$ of all urothelial cancers. During the last two decădês, the incidence of renal pelvic tumors has remained stable, although there has been a slight increase in the incidence of ureteral cancer (1). Recently, the distribution of upper tract urothelial carcinomas in the population has shifted to older patients, females, and non-white individuals. Similar to bladder carcinoma, smoking and occupational exposure to arylamines are well-established risk factors for upper tract urothelial carcinoma accounting for the majority of cases (2). There are also unusual exogenous carcinogenic factors unique to the upper urothelial tract, including analgesic nephropathy $(3,4)$ and Balkan nephropathy $(5,6)$. The 5-year cancer-specific survival for upper tract urothelial carcinoma in the United States approaches $75 \%$, and stage and grade are the most powerful predictors of survival (1). Nephroureterectomy with excision of the ipsilateral ureteral orifice and bladder cuff en bloc is the standard treatment, and conservative management has evolved through the necessity for renal preservation in some patients, particularly those with low-grade and low-stage tumors (7).

There is growing epidemiological and genetic evidence that some carcinomas of the upper urinary tract develop as a manifestation of the hereditary nonpolyposis colorectal cancer syndrome (HNPCC; 
8). This syndrome is characterized by germline mutations in a number of DNA mismatch repair genes, leading to tumor development in a number of organs and detectable as microsatellite instability in the DNA and loss of immunohistochemical staining for the DNA mismatch repair enzymes in tumor tissue $(9,10)$. Patients with HNPCC can develop a number of extracolonic tumors, including cancers of the endometrium, ovaries, stomach, and urinary tract, particularly urothelial carcinoma of the renal pelvis and ureter $(8,11)$. The aim of this study was to characterize the clinicopathological features, including cancer histories, in a well-defined cohort of patients with urothelial cancer of the upper urinary tract, and to determine the frequency of microsatellite instability in these upper tract urothelial carcinomas.

\section{MATERIAL AND METHODS}

\section{Patient Characteristics}

The study was approved by the Mayo Clinic Institutional Review Board. Patients had to have provided research authorization to be included in the study. The study cohort consisted of 114 consecutive patients surgically treated for urothelial carcinoma of the upper tract (renal pelvis $=61$ and ureter $=53$ ) between 1985 and 1992. Clinical and epidemiological data were obtained through chārt review without knowledge of the microsatellite status. Pathologic features including pathologic stage (International Union Against Cancer; 12), histologic grade (World Health Organization; 13) and growth architecture (papillary versus flat) were verified independently through microscopic slide review (AH, $\mathrm{HB}$ ). The patients were seen for regular outpatient visits for $\geq 7$ years, and/or were followed by yearly questionnaires sent to their primary physician.

\section{Molecular Analyses}

Matched normal and tumor DNA was extracted from paraffin-embedded tissue for the microsatellite analysis as described previously (14). In brief, $10-\mu \mathrm{m}$ histologic tissue sections were deparaffinized and stained with methylene blue for approximately 15 seconds. Tumor cell aggregates were separated from surrounding stromal cells by microdissection under an inverted microscope at $40 \times$ magnification. The microdissected tissue samples contained $\geq 80 \%$ tumor cells. Renal parenchyma without tumor infiltration, lamina propria, muscularis propria of the ureter, or adipose tissue surrounding ureter or renal pelvis served as the normal tissue sample. Microdissection using laser microdissection (PALM) was performed in cases where there was low tumor content, limited normal tissue, or a large amount of stromal and inflammatory cells relative to tumor (15). Microdissected tissue samples were digested overnight with a proteinase $\mathrm{K}$-containing lysis buffer, and the lysate was further processed using a standard column-based DNA purification kit (Qiagen, Hilden, Germany). Microsatellite analysis was performed using a panel of six microsatellite loci, including five markers of the reference panel recommended by the National Cancer Institute (16). In addition to the reference panel markers (BAT25, BAT26, D2S123, D5S346, and D17S250), the mononucleotide marker BAT40 was used, because in our experience, it is the most sensitive and specific for microsatellite instability among 31 markers used in colorectal cancer, and the primer sequences have been described previously (17). PCR amplifications were performed with $100 \mathrm{ng}$ of purified genomic DNA in a final volume of $20 \mu \mathrm{L}$ in an MJ Research Thermocycler (PTC100; MJ Research, Watertown, MA) and the polymerase chain reaction (PCR) products were electrophoresed through gels containing $6.7 \%$ polyacrylamide and $50 \%$ urea. Microsatellite instability was defined by the presence of novel bands after PCR amplification of tumor DNA that were not present in the PCR products of the corresponding normal DNA. Tumor samples were included in the microsatellite analysis only if at least five microsatellite loci could be analyzed. All gels were evaluated independently by two observers (AH, WD). A tumor was classified as microsatellite unstable if more than one of the six panel markers showed instability. Each paired sample was analyzed at least twice to verify the results.

\section{Statistical Analyses}

Cancer-specific survival was estimated by the method of Kaplan and Meier (18) and compared between patients with various tumor stages and histologic grades, and between patients with microsatellite-stable and -unstable tumors by the exact log-rank statistics (StatXact; 19). Because the number of deaths was relatively small $(n=33)$, exact $P$ values were computed for the log-rank statistics. The frequencies of events in the patient histories and family histories were compared by the Fisher exact test using the StatXact software package statistics (19). All $P$ values resulted from twosided tests.

\section{RESULTS}

\section{Clinicopathological Characteristics}

The study cohort consisted of 114 consecutive patients with surgically resected urothelial carcinoma of the upper urinary tract (renal pelvis and 
TABLE 1. Patients with Microsatellite Stable or Microsatellite Unstable Urothelial Carcinomas of the Upper Urinary Tract: Positive Family History of Cancer

\begin{tabular}{lcc}
\hline \multicolumn{1}{c}{ Group } & $\begin{array}{c}\text { One } \\
\text { First-Degree } \\
\text { Relative, } \\
n(\%)\end{array}$ & $\begin{array}{c}\text { Two or } \\
\text { More } \\
\text { First-Degree } \\
\text { Relatives, } \\
n(\%)\end{array}$ \\
\hline All patients $(n=114)$ & $66(58)$ & $17(15)$ \\
Microsatellite-stable tumors $(n=46)$ & $19(41)$ & $8(17)$ \\
Microsatellite-unstable tumors $(n=21)$ & $13(62)$ & $2(10)$ \\
\hline
\end{tabular}

ureter). Sixty-one $(53.5 \%)$ patients had urothelial carcinoma of the renal pelvis, and 53 (46.5\%) had urothelial carcinoma of the ureter. Eighty-four (73.7\%) patients were male, and 30 (26.3\%) were female (ratio 2.8:1). The median age was 70 years (range, 28 to 92 y). Eighty-three patients $(72.8 \%$ ) were smokers, with an average of $1.2 \pm 0.54$ packs per day and $34 \pm 13.3$ years of smoking. The malefemale ratio in smokers and nonsmokers was significantly different (7.3:1 and $0.55: 1$, respectively; $P$ $<$.0001). Clinicopathological features and the frequency of microsatellite instability did not differ between smokers and non-smokers. Sixty-six (57.9\%) patients had a positive family history of cancer (at least one first-degree relative affected with a malignancy), and 17 (14.9\%) patients had two or more first-degree relatives diagnosed with cancer. Only one patient was clinically recognized having HNPCC, but genetic testing had not been performed in any patient. The frequency of microsatellite instability did not differ among patients with one or more than one first-degree relative with cancer (Table 1). Only six (5.3\%) patients had a positive family history of urothelial cancer, and all six patients presented with microsatellite-stable tumors. The distribution of histologic grade, pathologic stage, and growth pattern is shown in Table 2. The distribution of time to death according to tumor location, histologic grade, and pathologic stage as estimated by the method of Kaplan and Meier (18) is shown in Fig. 1A-C.

\section{Microsatellite Analysis}

Microdissected tissue samples yielded amplifiable DNA for a complete microsatellite analysis

\begin{tabular}{|c|c|c|c|c|c|}
\hline \multirow{2}{*}{$\frac{\text { Variable }}{\text { Histologic Grade }}$} & \multicolumn{5}{|c|}{ Subcategory } \\
\hline & 1 & & 2 & & 3 \\
\hline & 19 & & 54 & & 41 \\
\hline \multirow[t]{2}{*}{ Pathologic Stage } & $\mathrm{Ta}$ & 1 & 2 & 3 & 4 \\
\hline & 68 & 13 & 10 & 21 & 2 \\
\hline \multirow[t]{2}{*}{ Growth } & & papilla & & solic & \\
\hline & & 96 & & 18 & \\
\hline
\end{tabular}

All data are $n$ from both tumor and normal tissue in 67 (58.8\%) patients. Unsuccessful microsatellite analysis in the remaining tumor samples was a result of the lack of sufficient amounts of normal tissue or failure to amplify at least five microsatellite loci. Clinicopathologic parameters including tumor location, gender, age, histologic grade, pathologic stage, and growth pattern did not differ between successfully amplified tumor samples and nonamplified tumors. Microsatellite analysis revealed 21 patients (31.3\%) with tumors that exhibited microsatellite instability (more than one of six consensus markers unstable in at least five successfully amplified loci; Fig. 2). Forty-six (68.7\%) patients had microsatellite-stable tumors. Among those 46, 11 patients had tumors that showed instability in only one of six markers. These patients were subsequently combined into the microsatellite-stable group for further analyses. The distribution of gender, age at diagnosis, smoking history, and location of tumor was not different between patients with microsatellite-stable and microsatellite-unstable urothelial carcinomas of the upper urinary tract. Likewise, survival time distributions were not significantly different between these two cohorts (Fig. 3 ). The number of patients with more than one additional cancer in their medical history was significantly greater in the microsatellite-unstable group compared with in the microsatellite-stable group ( $P=.015$; Table 3 ). Likewise, the number of patients with a history of colorectal carcinoma or family history for colorectal carcinoma was significantly greater in the microsatellite-unstable group compared with in the microsatellite-stable group $(P$ $=.001$ and $P=.026$, respectively; Table 3 ). Analysis of histologic grade and pathologic stage in patients with microsatellite-stable and microsatelliteunstable urothelial carcinomas of the upper urinary tract revealed significantly fewer patients with Grade 1 tumors in the microsatellite instability cohort $(P=.028$, Table 4$)$.

\section{DISCUSSION}

It is well known that tumor grade and stage in urothelial cancers of the upper urinary tract are powerful predictors of patient outcome (7). Grade and stage are closely related, and the vast majority of World Health Organization Grade 1 (of 3) urothelial carcinomas are noninvasive, whereas the majority of higher grade tumors show invasion into the lamina propria or muscle layer (7). Previous studies at the Mayo Clinic showed that survival in patients with low-grade urothelial cancers of the upper urinary tract is identical to age-matched controls (20), whereas survival of patients with higher grade tumors correlated with advanced stage and was sig- 


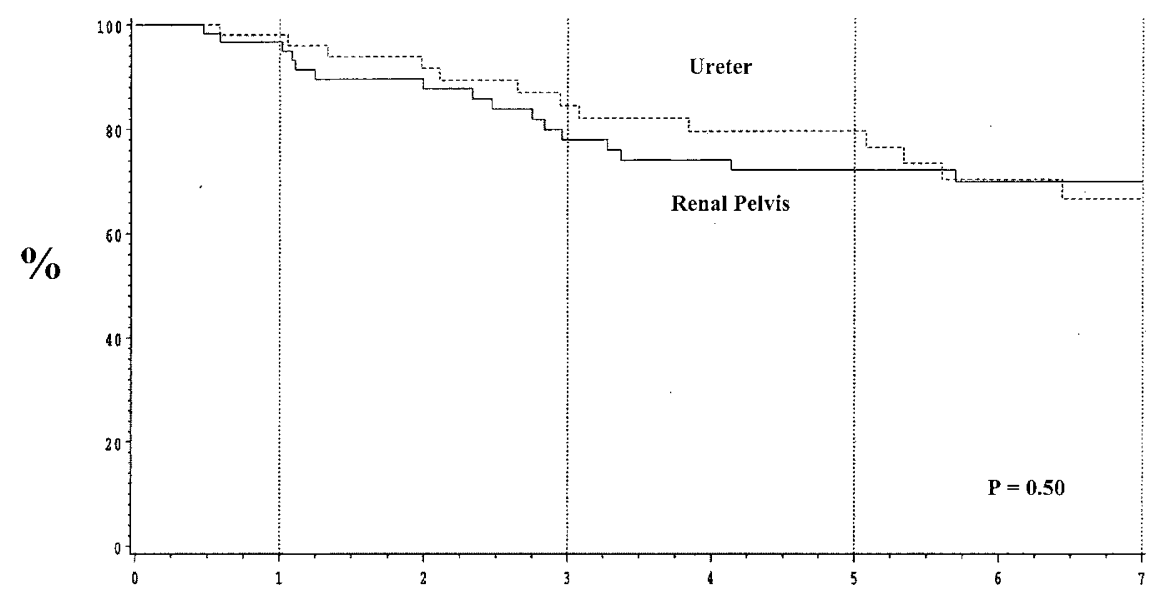

A

\section{Years}
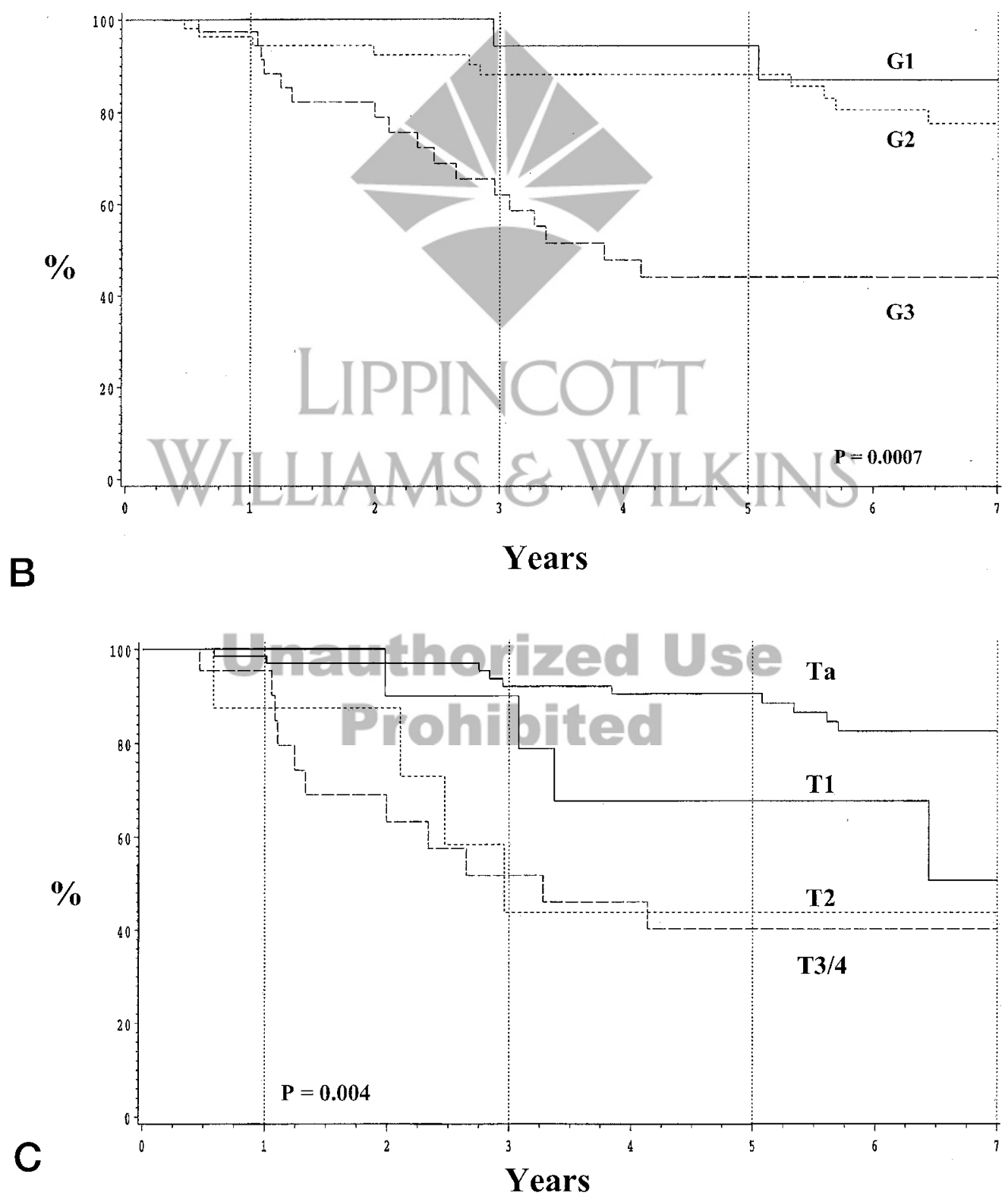

FIGURE 1. Distribution of time to death according to tumor location (A), histologic grade (B), and pathologic stage (C) among patients with urothelial carcinomas $(n=114)$ of the upper urinary tract as estimated by the method of Kaplan and Meier (18). 


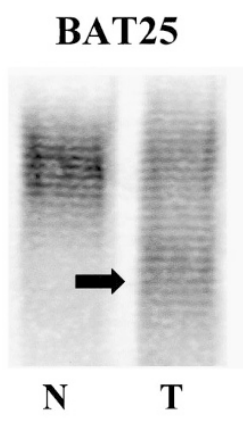

BAT26

BAT40

D2S123

D5S346

D17S250
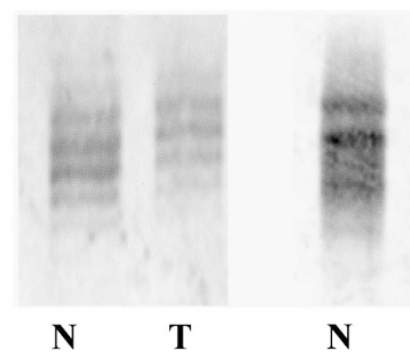

$\mathbf{N}$

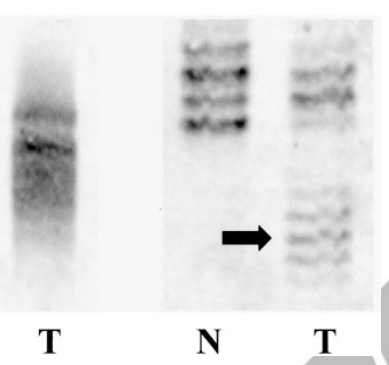

T

FIGURE 2. Analysis in a patient with a microsatellite-unstable tumor using six microsatellite markers (BAT25, BAT26, BAT40, D2S123, D5S346, D17S250). Arrows mark the backshifts in the tumor DNA in four of six investigated markers. N, normal DNA; T, tumor DNA.

nificantly lower than patients with low-grade tumors and control patients $(21,22)$. In our study, there was no difference in cancer-specific survival between patients with renal pelvic and ureteral cancers (Fig. 1A). Similarly, all other clinical and pathological parameters did not differ between patients with renal pelvic and ureteral tumors. Thus, our clinicopathological analysis was performed on the entire set of 114 patients. Our analysis confirms the survival differences related to histologic grade and pathologic stage (Fig. 1B-C). The cancerspecific survival according to pathologic stage showed a clear separation of the survival curves between patients with pTa tumors and pT1/T2 tumors, and pT3/4 tumors (Fig. 1C). The prognostic impact of invasive growth makes accurate pathologic staging critical to predicting patient outcome

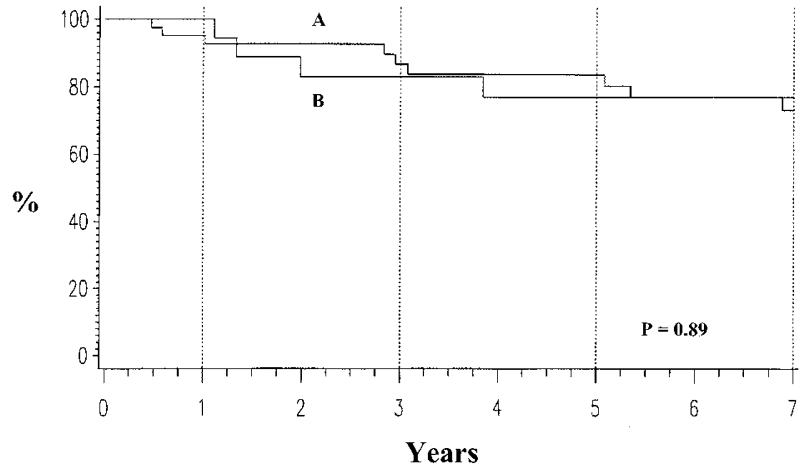

FIGURE 3. Distribution of time to death among patients with microsatellite-stable (A) and microsatellite-unstable (B) urothelial carcinomas of the upper urinary tract as estimated by the method of Kaplan and Meier (18).

as other modalities are not appropriate for staging (23-25).

Patients with HNPCC can develop carcinomas of multiple organs including the upper urinary tract. In the colon, HNPCC-related carcinomas occur in younger patients and are more commonly right sided, poorly differentiated, and with unusual histological characteristics, including increased numbers of intratumoral lymphocytes (26). Cancerspecific survival of patients with HNPCC-related carcinomas or carcinomas with microsatellite instability is better than that of patients with sporadic colorectal cancer when matched for stage $(27,28)$. In our study, we found a lower frequency of lowgrade urothelial cancers in patients with microsatellite instability, but there was no difference in either age or survival between patients with microsatellite-stable and microsatellite-unstable urothelial cancers. However, the study cohort is small, and analysis of survival and specific morphologic features of upper-tract urothelial carcinoma related to microsatellite instability and HNPCC will require additional analysis with larger numbers of patients. Interestingly, similar observations as ours have been made in patients with endometrial cancers and HNPCC (29). In other studies, microsatellite instability in urothelial carcinomas of the uri-

TABLE 3. Patients with Microsatellite-Stable or Microsatellite-Unstable Urothelial Carcinomas of the Upper Urinary Tract: Additional Lifetime Malignancies and Cancer History

\begin{tabular}{|c|c|c|}
\hline Variable & $\begin{array}{l}\text { Microsatellite-Stable Tumors } \\
\qquad(n=46), n(\%)\end{array}$ & $\begin{array}{l}\text { Microsatellite-Unstable Tumors } \\
\qquad(n=21), n(\%)\end{array}$ \\
\hline \multicolumn{3}{|c|}{ No. additional lifetime malignancies } \\
\hline Urothelial cancer only & $12(26)$ & $3(14)$ \\
\hline One & $11(24)$ & $4(19)$ \\
\hline Two or more & $4(9)$ & $7(33)^{*}$ \\
\hline \multicolumn{3}{|c|}{ Positive colon cancer history } \\
\hline Family & $5(11)$ & $7(33)^{* *}$ \\
\hline Own & $1(2)$ & $6(29)^{* * *}$ \\
\hline
\end{tabular}

\footnotetext{
* $P=.015$.

** $P=.026$.

$* * * P=.001$
} 
TABLE 4. Distribution of Histologic Grade and Pathologic Stage in Patients With Microsatellite-Stable and Microsatellite-Unstable Urothelial Carcinomas of the Upper Urinary Tract

\begin{tabular}{lcc}
\hline Variable & $\begin{array}{c}\text { Microsatellite-Stable } \\
\text { Tumors } \\
(n=46), n(\%)\end{array}$ & $\begin{array}{c}\text { Microsatellite-Unstable } \\
\text { Tumors } \\
(n=21), n(\%)\end{array}$ \\
\hline Histologic grade & & \\
1 & $13(28)$ & $1(5)^{a}$ \\
2 & $21(46)$ & $13(62)$ \\
3 & $12(26)$ & $7(33)$ \\
Pathologic stage & & \\
Ta & $33(72)$ & $14(66)$ \\
1 & $5(11)$ & $1(5)$ \\
2 & $5(11)$ & $2(10)$ \\
3 & $3(6)$ & $4(19)$ \\
4 & 0 & 0 \\
\hline$a P=.028$. & &
\end{tabular}

nary bladder is reported to be rare in superficial tumors but more common in invasive tumors (30). However, we did not find a difference in the distribution of pathologic stage in between patients with microsatellite-stable and microsatellite-unstable tumors.

At least two types of genetic instability have been demonstrated in cancer cells; one characterized by a high frequency of chromosomal aberrations and the other by mutations in DNA sequences resulting from defects in the DNA mismatch repair system and associated with microsatellite instability (31). The DNA mismatch repair system provides normal cells with a high level of protection against mutations arising during DNA replication. It consists of several proteins encoded by multigene families, consisting of hMSH2, hMSH3, hMSH6, and hMLH1, hPMS1, and hPMS2 (16). Their inactivation results in a large increase in spontaneous mutability and a direct oncogenic effect with the expression of a "mutator phenotype" (32). Microsatellite instability is reflected in alterations in the patterns of polymorphic, short, tandem-repeat segments (microsatellites) dispersed throughout the human genome. Studies indicate that microsatellite instability reflects an underlying genomic instability resulting from inactivation of both alleles at a DNA mismatch repair gene locus $(9,33)$. Some urothelial carcinomas develop as a manifestation of HNPCC, characterized by germline mutations in a number of these DNA mismatch repair genes. There is a 14 -fold-increased risk of urothelial cancer of the renal pelvis and ureter in patients with HNPCC (8). Previous studies of microsatellite instability in bladder cancer have shown mutation frequencies that are generally very low (3335). Because sporadic tumors of the renal pelvis, ureter, and bladder share the same risk factors, it is unclear why the risk of bladder cancer is not increased in patients with HNPCC. Similarly it is unclear why patients with HNPCC develop carcinoma at a few specific organ sites or why microsatellite insta- bility unrelated to HNPCC is more common in certain tumor types. Interestingly, the frequency of microsatellite instability in our study (31.3\%) is the highest reported to date in a cohort of consecutive surgically treated patients with urothelial carcinoma of the upper urinary tract. Differences in the reported frequencies of microsatellite instability in various tumors may be in part caused by methodological differences. Studies may examine patient cohorts that differ in their distribution of grade and stage, or the panel of microsatellites selected may differ. Recent descriptions of microsatellite instability in other extracolonic cancers have also differed significantly from earlier reports partly because of the interpretation and definition of microsatellite instability. Our study involved consecutive patients, and a selection bias toward various grades and stages seems unlikely. The successful DNA preparation and amplification of microsatellite loci in only 67 of 114 tumor samples is unlikely to have influenced our results because all tumor and patient characteristics in amplified and not amplified tumor samples were not different. Because of the clinical importance of the microsatellite assay, a number of guidelines recently have been introduced for improving its reliability (16), including suggested reference panel markers. Using the reference panel, microsatellite instability is defined as having instability in two ore more markers. Tumors with only one unstable repeat are considered not to have microsatellite instability because it may be a chance occurrence and not a result of a mutation in a DNA repair gene (36). In addition to problems in the selection of DNA markers, other parameters can obscure the accurate evaluation of a tumor sample for microsatellite instability, such as large amount of normal cells in tumor samples, poor DNA preparation, and variations in the interpretation of gel band patterns.

Male patients predominated in our study cohort of consecutive patients, with a male-female ratio of 2.8:1, suggesting as has been demonstrated in previous studies that cigarette smoking is a significant contributor to the development of upper tract urothelial carcinoma. The complex mixture of cancer initiators and promotors in cigarette smoke, such as $N$-nitrosamines, polycyclic aromatic amines, and benzo(a)pyrene are thought to be important exogenous factors in the carcinogenesis of urothelial carcinoma $(37,38)$. The number of smokers in the studied cohort (73\%) was high, with a substantial average amount of pack-years, and this supports the known association of smoking and tumors of the urinary tract. Accordingly, the malefemale ratio in smokers and nonsmokers differed significantly (7.3: 1 and 0.55: 1, respectively). Smoking increases the risk of both bladder cancer and upper urinary tract cancers (39), and active smokers have an approximately 3 -fold higher risk of urinary tract cancer than nonsmokers, whereas the risk for 
former cigarette smokers remains 2-fold elevated (40). Uchida et al. (30) reported a somewhat higher rate of microsatellite instability in tumors from smokers. However, no relationship of cigarette smoking with microsatellite instability was found in our study.

We found that $58 \%$ of all patients to have at least one first-degree relative with cancer, and $15 \%$ of patients had two or more first-degree relatives with cancer. These rates did not differ between patients with microsatellite-unstable tumors and patients with microsatellite-stable tumors (Table 1). There were significantly more patients in the microsatellite-unstable group who had two or more additional malignancies, whereas the rates of only one additional neoplasm and the prevalence of additional urothelial cancers did not differ between microsatellite-unstable and microsatellite-stable patients (Table 3). In contrast, the rate of colon cancer in either the patient or family was significantly greater in patients with microsatellite instability (Table 3).

The two major causes of urothelial cancer, cigarette smoking, and occupational exposure to arylamines, have been recognized for 4 decades (2). Other environmental risk factors unique to the upper urothelial tract, such as analgesic nephropathy $(3,4)$ and Balkan nephropathy $(5,6)$, have been identified, and recent reports point to polluted artesian well water (41) and Chinese herbs (42) as causative agents. Comparatively little is known about genetic predispositions in urothelial cancers (43). Numerous case reports document the clustering of urothelial carcinoma in families (44), several of which demonstrate an extremely early age of onset of disease, but the underlying genetic mechanisms remain elusive (45). Our study showed a high frequency of microsatellite instability (31.3\%) in consecutive surgically treated upper urothelial tract carcinomas, the highest frequency reported to date, highlighting such tumors as an important part in the clinical spectrum of microsatellite instability and HNPCC. In such patients, the cumulative risk for urothelial carcinomas of the upper urinary tract is relatively low, although a subset of HNPCC families may be exposed to a much higher risk. Despite recent advances in the understanding of the molecular genetic basis of HNPCC, it remains difficult to identify patients with HNPCC before the development of malignancies. As yet nothing is known of the clinical impact of screening patients with upper tract urothelial carcinoma for HNPCC. Likewise, the potential clinical benefit of screening for upper urinary tract cancers in patients with the HNPCC syndrome is unknown. Certainly, patients who develop colorectal cancer and upper tract urothelial carcinoma might be candidates to be screened for the possibility of HNPCC.
Our findings reinforce the importance of obtaining comprehensive patient and family histories of cancer in patients with urothelial carcinoma of the renal pelvis and ureter. Subsequent identification of HNPCC individuals enables the patient and atrisk relatives to benefit from targeted surveillance and management programs.

\section{REFERENCES}

1. Munoz JJ, Ellison LM. Upper tract urothelial neoplasms: incidence and survival during the last 2 decades. J Urol 2000;164:1523-5.

2. Ross RK, Jones PA, Yu MC. Bladder cancer epidemiology and pathogenesis. Semin Oncol 1996;23:536-45.

3. Mihatsch MJ, Zollinger HU, Torhorst J. Pathognomonic lesion of analgesic nephropathy. N Engl J Med 1979;300:1275-6.

4. Stewart JH, Hobbs JB, McCredie MRE. Morphologic evidence that analgesic-induced kidney pathology contributes to the progression of tumors of the renal pelvis. Cancer 1999;86: 1576-82.

5. Petkovic SD. Epidemiology and treatment of renal pelvic and ureteral tumors. J Urol 1975;114:858.

6. Radovanovic Z, Krajinovic S, Jancovic S, et al. Family history of cancer among cases of upper urothelial tumours in a Balkan nephropathy area. J Cancer Res Clin Oncol 1985;110: 181.

7. Tawfiek ER, Bagley DH. Upper-tract transitional cell carcinoma. Urology 1997;50:321-9.

8. Sijmons RH, Kiemeney LALM, Witjes JA, et al. Urinary tract cancer and hereditary nonpolyposis colorectal cancer: risks and screening options. J Urol 1998;160:466-70.

9. Bocker T, Rüschoff J, Fishel R. Molecular diagnostics of can-

cer predisposition: hereditary non-polyposis colorectal carcinoma and mismatch repair defects. Biochim Biophys Acta 1999;1423:01-10.

10. Lynch HT, Smyrk T. Hereditary nonpolyposis colorectal cancer (Lynch syndrome). An updated review. Cancer 1996;78: 1149-67.

11. Watson P, Lin KM, Rodriguez-Bigas AM, et al. Colorectal carcinoma survival among hereditary nonpolyposis colorectal carcinoma family members. Cancer 1998;83:259-66.

12. Sobin LH, Wittekind CH, editors. TNM classification of malignant tumors. 5th ed. New York: Wiley-Liss; 1997.

13. Mostofi FK, Davis CJ, Sesterhenn IA, editors. Histological typing of urinary bladder tumors. 2nd ed. Berlin: Springer; 1999.

14. Rüschoff J, Dietmaier W, Luttges J, et al. Poorly differentiated colonic adenocarcinoma, medullary type: clinical, phenotypic, and molecular characteristics. Am J Pathol 1997;150: 1815-25.

15. Schütze K, Lahr G. Laser micromanipulation systems as universal tools in molecular biology and medicine. J Cell Mol Biol 1998;14:735-46.

16. Boland CR, Thibodeau SN, Hamilton SR, et al. A National Cancer Institute workshop on microsatellite instability for cancer detection and familial predisposition: development of international criteria for the determination of microsatellite instability in colorectal cancer. Cancer Res 1998;58:5248-57.

17. Dietmaier W, Wallinger S, Bocker T, et al. Diagnostic microsatellite instability: definition and correlation with mismatch repair protein expression. Cancer Res 1997;57:4749-56.

18. Kaplan EL, Meier P. Nonparametric estimation from incomplete observation. J Am Stat Assoc 1958;53:457-81.

19. Anonymous. Stat Xact [computer program]. Cambridge, MA: Cytel Software Corporation; 1991. 
20. Murphy DM, Zincke H, Furlow WL. Primary grade 1 transitional cell carcinoma of the renal pelvis and ureter. J Urol 1980;123:629-31.

21. Elliot DS, Blute ML, Patterson DE, et al. Long-term follow-up of endoscopically treated upper urinary tract transitional cell carcinoma. Urology 1996;47:819-25.

22. Murphy DM, Zincke H, Furlow WL. Management of high grade transitional cell carcinoma of the upper urinary tract. J Urol 1981;125:25-9.

23. Badalament RA, Bennett WF, Bova JG, et al. Computed tomography of primary transitional cell carcinoma of upper urothelial tracts. Urology 1992;40:71-5.

24. McCoy JG, Honda H, Reznicek M, et al. Computerized tomography for detection and staging of localized and pathologically defined upper tract urothelial tumors. J Urol 1991;146:1500-3.

25. Huffman JL, Bagley DH, Lyon ES, et al. Endoscopic diagnosis and treatment of upper tract urothelial tumors. A preliminary report. Cancer 1985;55:1422-8.

26. Jass JR. Pathology of hereditary nonpolyposis colorectal cancer. Ann NY Acad Sci 2000;910:62-73.

27. Gryfe R, Kim H, Hsieh ETK, et al. Tumor microsatellite instability and clinical outcome in young patients with colorectal cancer. N Engl J Med 2000;342:67-77.

28. Watson P, Lynch HT. Extracolonic cancer in hereditary nonpolyposis colorectal cancer. Cancer 1993;71:677-85.

29. Basil JB, Goodfellow PJ, Rader JS, et al. Clinical significance of microsatellite instability in endometrial carcinoma. Cancer 2000;89:1758-64.

30. Uchida T, Wang C, Wada C, et al. Microsatellite instability in transitional cell carcinoma of the urinary tract and its relationship to clinicopathological variables and smoking. Int J Cancer (Pred Oncol) 1996;69:142-5.

31. Lengauer C, Kinzler KW, Vogelstein B. Genetic instabilities in human cancers. Nature 1998;396:643-9.

32. Loeb LA. A mutator phenotype in cancer. Cancer Res 2001; 61:3230-9.
33. Arzimanoglou II, Gilbert F, Barber HRK. Microsatellite instability in human solid tumors. Cancer 1998;82:1808-20.

34. Bonnal C, Ravery V, Toublanc M, et al. Absence of microsatellite instability in transitional cell carcinoma of the bladder. Urology 2000;55:287-91.

35. Takahashi T, Kakehi Y, Mitsumori K, et al. Distinct microsatellite alterations in upper urinary tract tumors and subsequent bladder tumors. J Urol 2001;165:672-7.

36. Papadopoulos N. Microsatellite instability (MSI) in noncolonic, non-HNPCC tumors: "instable" evidence? Ann Oncol 1999;10:751-2.

37. Leistikow BN. The human and financial costs of smoking. Clin Chest Med 2000;21:189-97.

38. Tominaga S. Major avoidable risk factors of cancer. Cancer Lett 1999;143(Suppl 1):S19-23.

39. Pommer W, Bronder E, Klimpel A, et al. Urothelial cancer at different tumour sites: role of smoking and habitual intake of analgesics and laxatives. Results of the Berlin Urothelial Cancer Study. Nephrol Dial Transplant 1999;14:2892-7.

40. Zeegers MP, Tan FE, Dorant E, et al. The impact of characteristics of cigarette smoking on urinary tract cancer risk: a meta-analysis of epidemiological studies. Cancer 2000;89: $630-9$.

41. Chou YH, Huang CH. Unusual clinical presentation of upper urothelial carcinoma in Taiwan. Cancer 1999;85:1342-4.

42. Nortier JL, Martinez MC, Schmeiser HH, et al. Urothelial carcinoma associated with the use of a Chinese herb. N Engl J Med 2000;342:1686-92.

43. Aben KKH, Kiemeney LALM. Epidemiology of bladder cancer. Eur Urol 1999;36:1-13.

44. Kiemeney LALM, Schoenberg M. Familial transitional cell carcinoma. J Urol 1996;156:867-72.

45. Linn JF, Sesterhenn I, Mostofi FK, et al. The molecular characteristics of bladder cancer in young patients. J Urol 1998; 159:1493-6.
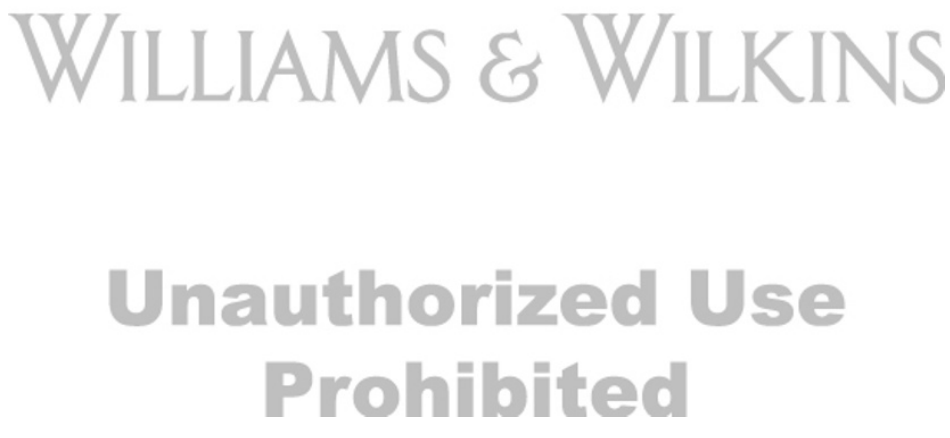